\title{
CHARACTERIZATION OF STRICTLY CONVEX DOMAINS BIHOLOMORPHIC TO A CIRCULAR DOMAIN
}

\author{
BY GIORGIO PATRIZIO ${ }^{1}$
}

Recently parabolic exhaustions have been used successfully to classify complex spaces (Stoll [6 and 7], Burns [2], P. Wong [9]). Here we define parabolic exhaustions for strictly convex domains in $\mathbf{C}^{n}$ and give necessary and sufficient conditions for such a domain to be biholomorphically equivalent to a circular domain or even to the ball.

The results presented in this note are part of the $\mathrm{Ph} . \mathrm{D}$. Thesis the author is completing under the direction of Professor W. Stoll. Details and more implications will appear at a later date.

A strictly convex domain $D \subset \mathbf{C}^{n}$ is a domain for which there exists a defining function whose real Hessian is strictly positive on $T_{x}(\partial D)$ for all $x \in \partial D$. Let $\Delta$ be the open unit disk in $\mathbf{C}$ and let $S$ be the unit sphere in $\mathbf{C}^{n}$. Let $D \subset \mathbf{C}^{n}$ be a strictly convex domain and $p \in D$ be any point. For $b \in S$ Lempert [4] constructs an extremal map $F(\square, b): \Delta \rightarrow D$ which is holomorphic with $F(0, b)=p$ and $F^{\prime}(0, b)=\lambda$ where $1 / \lambda>0$ is the length of $b$ in the infinitesimal Kobayashi metric of $D$ at $p$. These conditions determine $F(\square, b)$ uniquely and the map extends smoothly to an embedding $F(\square, b): \bar{\Delta} \rightarrow \bar{D}$. Also $F: \bar{\Delta} \times S \rightarrow \bar{D}$ is of class $C^{\infty}$ and surjective. One and only one function $\tau: \bar{D} \rightarrow \mathbf{R}_{+}$exists such that $\tau(F(z, b))=|z|^{2}$ for $(z, b) \in \bar{\Delta} \times S$. Then $\tau$ is a continuous exhaustion of $\bar{D}$, positive and of class $C^{\infty}$ on $\bar{D} \backslash\{p\}$. Also $\tau \equiv 1$ on $\partial D$. We refer to $\tau$ as the Lempert exhaustion of $D$ at $p$.

THEOREM 1. The Lempert exhaustion at $p$ is strictly parabolic on $D_{*}=$ $D \backslash\{p\}$, which means that on $D_{*}$
(1) $d d^{c} \tau>0$
(2) $d d^{c} \log \tau \geq 0$,
(3) $\left(d d^{c} \log \tau\right)^{n} \equiv 0$.

Properties (2) and (3) were proved by Lempert. For each $r$ with $0<r<1$ the pseudoball $D(r)=\left\{x \in D \mid \tau(x)<r^{2}\right\}$ is a ball in the Kobayashi distance. By an argument of harmonic functions, it is shown that the Hessian of the defining function $\tau-r^{2}$ of $D(r)$ is positive on the tangent space of $\partial D(r)$ at every point of $\partial D(r)$. Hence $D(r)$ is strictly convex and $d d^{c} \tau>0$ follows easily.

The strictly parabolic function $\tau$ defines a Monge-Ampère foliation on $D$ which coincides with the foliation defined by $F$. More precisely if

$$
X=X^{\mu} \frac{\partial}{\partial z^{\mu}}=\tau_{\bar{\nu}} \tau^{\bar{\nu} \mu} \frac{\partial}{\partial z^{\mu}}
$$

Received by the editors November 18, 1982.

1980 Mathematics Subject Classification. Primary 32H99; Secondary 32F15.

${ }^{1}$ The author was supported by a grant from the Consiglio Nazionale delle Ricerche. 
is the complex gradient vector field associated to $\tau$ (see Stoll [6]), then we have

Proposition 2. If $b \in S$ and $0 \neq z \in \Delta$, then $X(F(z, b))=z F^{\prime}(z, b)$.

Since $F^{\prime}(0, b) \neq 0$ for all $b \in S$, a smooth, complete circular domain $G$ is given by

$$
G=\left\{z b|z \in \mathbf{C}, b \in S,| z \mid<\left\|F^{\prime}(0, b)\right\|\right\} .
$$

A homeomorphism $h: \bar{G} \rightarrow \bar{D}$, called the circular representation of $D$ at $p$, is defined by

$$
h(z c)=F\left(z, \frac{c}{\|c\|}\right)
$$

for all $z \in \bar{\Delta}$ and $c \in \partial G$. The map $h$ is of class $C^{\infty}$ on $G_{*}=G \backslash\{0\}$ with $h(0)=p$. It is holomorphic along each disk through the origin and if $M$ is the Minkowski functional of $G$, then $\tau \circ h=M^{2}$.

Proposition 3. The map $h: G \rightarrow D$ is biholomorphic iff $h$ is of class $C^{\infty}$ at 0 .

Proposition 4. Let $D_{j}$ be strictly convex domains for $j=1,2$. Let $h_{j}: G_{j} \rightarrow D_{j}$ be the circular representation of $D_{j}$ at $p_{j} \in D_{j}$. If $\phi: D_{1} \rightarrow D_{2}$ is a biholomorphic map with $\phi\left(p_{1}\right)=p_{2}$, then $\psi=h_{2}^{-1} \circ \phi \circ h_{1}: G_{1} \rightarrow G_{2}$ is biholomorphic and in fact is the $\mathbf{C}$-linear map $d \phi\left(p_{1}\right)$ restricted to $G_{1}$.

Thus the isotropy group of $\operatorname{Aut}(D)$ at $p$ lifts to a group of linear automorphisms of $G$.

The Monge-Ampère foliation is holomorphic if and only if $X$ is a holomorphic vector field.

THEOREM 5. The Monge-Ampère foliation associated to the Lempert exhaustion $\tau$ is holomorphic iff the circular representation map is biholomorphic.

It is most interesting to find conditions which force the Monge-Ampère foliation to be holomorphic. A one parameter group $\alpha: \mathbf{R} \times D \rightarrow D$ of automorphisms of $D$ is said to be uniform at $p \in D$ if $\alpha(t, p)=p$ for all $t \in \mathbf{R}$, $d \dot{\alpha}(0, p) \zeta=\lambda \zeta$ for all $\zeta \in \mathbf{C}^{n}$ and some constant $\lambda \in \mathbf{C}$. Here $\dot{\alpha}=\partial \alpha / \partial t$. If $\alpha$ is so given, let $Y$ be the associated vector field, $Y(q)=\dot{\alpha}(0, q)$ for $q \in D$. Then $\alpha$ lifts to a one parameter group of automorphisms of $G$ uniform at 0 . Also the vector field $X$ lifts to $\tilde{X}=z^{\mu} \partial / \partial z^{\mu}$ which enables us to show that $X=c Y$ for some constant $c$. Since $Y$ is holomorphic, so is $X$. By Theorem 5 we conclude

THEOREM 6. $D$ is biholomorphic to a circular domain iff there exists a one parameter group of automorphisms of $D$ uniform at some point of $D$.

This theorem generalizes a classical result in two variables of $\mathrm{H}$. Cartan [3].

We say that $D$ is rotational at $p \in D$ iff there is a one parameter group of automorphisms of $D$ uniform at $p$. Thus a strictly convex domain is biholomorphic to a circular domain iff it is rotational at least at one point. Combining results of Braun, Kaup and Upmeier [1], Rosay [5] and B. Wong [8], we obtain 
TheOREM 7. A strictly convex domain is biholomorphic to the ball iff it is rotational at least at two points.

\section{REFERENCES}

1. R. Braun, W. Kaup and H. Upmeier, On the automorphisms of circular and Reinhardt domains in complex Banach spaces, Manuscripta Math. 25 (1978), 97-133.

2. D. Burns, Curvature of Monge-Ampère foliations and parabolic manifolds, Ann. of Math. (2) 115 (1982), 349-373.

3. H. Cartan, Les fonctiones de deux variables complexes et le probleme de la représentation analitiques, J. Math. Pures Appl. 96 (1931), 1-114.

4. L. Lempert, La métrique de Kobayashi et la représentation des domaines sur la boule, Bull. Soc. Math. France 109 (1981), 427-474.

5. J. P. Rosay, Sur une characterization de la boule parmi les domaines de $\mathbf{C}^{n}$ par son group d'automorphismes, Ann. Inst. Fourier (Grenoble) 29 (1979), 91-97.

6. W. Stoll, The characterization of strictly parabolic manifolds, Ann. Scuola Norm. Sup. Pisa Cl. Sci. (4) 7 (1980), 87-154.

7. __ The characterization of strictly parabolic spaces, Compositio Math. 44 (1981), 305373.

8. B. Wong, Characterization of the ball in $\mathbf{C}^{n}$ by its automorphism group, Invent. Math. $\mathbf{4 1}$ (1977), 253-257.

9. P. M. Wong, Geometry of the homogeneous complex Monge-Ampère equation, Invent. Math. 67 (1982), 261-274.

Department of Mathematics, University of Notre Dame, Notre Dame, Indiana 46556 
